Archéologie du passé, mélancolie du présent - I

\title{
Souvenirs du réel. Remarques sur la mélancolie et le présent
}

\section{Andrea Cavazzini}

\section{OpenEdition \\ Journals}

Édition électronique

URL : http://journals.openedition.org/grm/1134

DOI : $10.4000 / \mathrm{grm} .1134$

ISSN : 1775-3902

\section{Éditeur}

Groupe de Recherches Matérialistes

\section{Référence électronique}

Andrea Cavazzini, «Souvenirs du réel. Remarques sur la mélancolie et le présent », Cahiers du GRM [En ligne], 13 | 2018, mis en ligne le 02 décembre 2018, consulté le 20 avril 2019. URL : http:// journals.openedition.org/grm/1134; DOI : 10.4000/grm.1134

Ce document a été généré automatiquement le 20 avril 2019

(c) GRM - Association 


\title{
Souvenirs du réel. Remarques sur la mélancolie et le présent
}

\author{
Andrea Cavazzini
}

\section{I}

1 Une certaine paresse intellectuelle associe la mélancolie au regret stérile du passé : c'est une lecture hâtive des derniers textes de Walter Benjamin, qui rappelait au contraire, après Péguy, que la fixation douloureuse au passé, paralysant la volonté et l'action, vient initialement d'une volonté et d'une action ici et maintenant défaillantes.

2 L'affect mélancolique est originairement lié à une perte inobjectivable, et aux manières de la combler à travers des ersatz : son noyau traumatique n'est pas un moment révolu, mais un objet perdu dans le creux duquel s'engouffrent des objets substitutifs. Ce n'est donc pas sans signification qu'un diagnostic portant sur des mobilisations politiques récentes, plus précisément sur la séquence qui commence avec Occupy Wall Street et qui finit provisoirement avec la " Nuit débout », caractérise ces épisodes à travers l'idée d'une absence et d'une compensation :

La communauté est devenue avant tout un objet de désir. C'est le phénomène

marquant du mouvement des places et des occupations ${ }^{1}$.

Jacques Rancière indique aussi d'une manière assez précise, bien qu'à travers un langage et un appareil conceptuel très particuliers, ce qui fait défaut à la scène où un tel désir vient évoquer des objets absents :

On a pu constater (...) à quel point [un peuple autre que celui du processus

électoral] est actuellement objet de désir plus que forme en mouvement. On sait en

figurer l'exigence mais on ne sait pas quels organes et quelles formes donner à sa constitution ${ }^{2}$.

On peut considérer des expressions telles que "forme en mouvement ", " organes", " constitution », comme des symptômes tenant lieu des termes traditionnels de l'ancien discours marxiste : l'organisation, la tactique, la stratégie...

3 Ces symptômes font allusion à une problématique que l'œuvre tout entière de Jacques Rancière, et plus en général la réflexion «radicale» post-marxiste, n'abordent pas 
fréquemment : la problématique de l'efficace, de la prise sur le réel. Les mouvements dont parle Jacques Rancière souffrent d'une incapacité structurelle de (se) réaliser, d'opérer une transformation du monde qui serait vérifiable par-delà les intentions et les énonciations. C'est cette incapacité que le remplacement de la "forme en mouvement » par le « désir » à la fois dénonce comme un manque et fige dans l'autosuffisance des simulacres évoqués par ce même désir :

Sur la place de la République, comme à Liberty Plaza ou à la Puerta del Sol, la centralité de la forme assemblée a montré en même temps la puissance d'un désir de communauté et d'égalité mais aussi la façon dont ce désir s'inhibe lui-même et s'enferme dans sa propre image, dans la mise en scène du bonheur d'être ensemble ${ }^{3}$

Il y a donc une ambivalence structurelle dans les pratiques des assemblées et des occupations : d'une part, de telles pratiques incarnent une demande visant la constitution d'un processus politique effectif; d'autre part, ces mêmes pratiques deviennent l'ersatz de cette constitution et de processus qui n'arrivent pas à s'inscrire dans un agencement déterminé d'étapes, de moyens et d'objectifs -, elles finissent ainsi par fournir une satisfaction imaginaire et intransitive à un désir désorienté :

Ce ne sont plus les usines ni mêmes les universités qui sont occupées, non plus les lieux de fonctions sociales mettant en présence des forces en conflit, mais l'espace vacant des places où la communauté se symbolise en assemblée aux temps de parole égalitaires tandis que dans les rues voisines résonnent des slogans comme « Tout le monde déteste la police » et que la destruction de quelques distributeurs de billets de banque compense dérisoirement la destruction de milliers d'emplis par des puissances financières contre lesquelles la lutte ouvrière s'est montrée impuissante ${ }^{4}$.

4 Autrement dit, les formes qui expriment le désir de « quelque chose d'autre » deviennent des images illusoires de l'objet de ce désir, et finissent ainsi par le couper de tout accès à une altérité réelle. Les militants et les activistes, les sujets qui soutiennent ces pratiques jouissent de la mise en scène de leurs aspirations, et s'enferment dans l'illusion que la politique, voire la " révolution », serait un travail de soi sur soi, de la pure et simple autoaffection dans laquelle la transformation du monde passerait par, et se réduirait à, écouter et voir les paroles et les gestes de son désir.

\section{II}

5 Toute une série de pratiques contemporaines oscilleraient donc entre l'expression d'une recherche, d'une quête, donc d'un manque, et la compensation imaginaire de ce manque. Mais qu'est-ce qui manque réellement dans ce désir? Qu'est-ce que ces formations imaginaires - slogans, assemblées, rassemblements belliqueux ou festifs - viennent substituer?

6 La réponse de Jacques Rancière est que ces formes tiennent lieu d'un "peuple» actuellement absent. Mais ce " peuple » reste aussi indéterminé que ses ersatz : il est luimême une formation imaginaire qui à la fois empêche et permet la formulation d'autre chose, donc d'un autre manque ${ }^{5}$. Cette multiplication des objets-écrans, venant se loger dans le vide d'une absence impossible à déterminer, fait l'objet de la méditation de Giorgio Agamben sur l'acedia, cet ancêtre théologique de la mélancolie moderne :

La mélancolie est moins une réaction de régression devant la perte de l'objet aimé qu'une aptitude fantasmatique à faire apparaître comme perdu un objet qui échappe à l'appropriation ${ }^{6}$. 
Autrement dit, « dans la mélancolie non seulement il est difficile de préciser ce qui a été perdu, mais il n'est même pas certain que l'on puisse parler de perte » :

[Selon Freud], la mélancolie est une réaction à la perte d'un objet d'amour, à laquelle cependant ne fait pas suite (...) un transfert de la libido sur un nouvel objet, mais son retrait dans le moi, narcissiquement identifié à l'objet perdu .

Le « peuple » n'est donc pas l'objet réel absent dont la perte déclencherait un désir teinté de mélancolie ; il est en réalité l'objet fictif engendré par le reflux de la libido vers le sujet désirant. C'est l'incapacité des formes de ce désir à sortir d'une satisfaction purement immanente qui fait apparaître le "peuple» en tant qu'ersatz-écran d'un manque inexprimable : ce qui rend en même temps difficile de saisir la vraie source de ce manque tel qu'il trouve son expression dans les assemblées et les occupations.

8 Giorgio Agamben a pourtant essayé de fournir une lecture dialectique du processus mélancolique. Il rappelle que « dans la mélancolie l'objet n'est ni approprié ni perdu mais approprié et perdu simultanément » :

En recouvrant son objet des noires tentures du deuil, la mélancolie lui confère la fantasmagorique réalité de l'objet perdu; mais dans la mesure où elle est le deuil d'un objet insaisissable, sa stratégie permet à l'irréel d'accéder à l'existence, délimitant une scène sur laquelle le moi peut entrer en rapport avec lui et tenter une appropriation qu'aucune possession ne pourrait égalers.

Cette lecture a le mérite de rappeler que la mélancolie a affaire moins à la perte d'un objet qu'à la prolifération illimitée d'objets substitutifs se logeant dans la béance d'une perte sans objet (du moins sans objet déterminable de la part du sujet). Il y a une productivité de la mélancolie, que l'on constate dans la multiplicité des formations substitutives qu'elle engendre.

Un autre point que l'analyse d'Agamben rappelle à juste titre est que la production objectale mélancolique permet malgré tout à un sujet de tenir : elle institue un rapport relativement maîtrisable avec une négativité autrement insurmontable, tout en permettant de situer dans un objet ce manque qui est plus proprement la blessure intime du sujet.

Dans ce jeu de remplacements et de déplacements, l'annihilation définitive du sujet est évitée, mais au prix d'un accès barré au réel : le sujet ne dispose plus, pour conserver sa consistance, que la delectatio morosa procurée par la consommation indéfinie d'objets qui ne sont que le prolongement imaginaire du moi.

La mélancolie est nécessairement associée à la tendance à rechercher son salut en soimême - ce qui est finalement la perte radicale de l'espérance, si l'on entend par ce mot une vertu qui «ne va pas de soi », selon l'expression de Péguy, car elle vise ce qui est audelà de notre savoir et de notre savoir-faire actuels.

La perte ineffable n'est pas la perte de soi - acception vulgaire de l'idée d'aliénation mais la perte d'un rapport à un " autre » irréductible, à la résistance et à la consistance d'un hétérogène qui dépasse la sphère immédiate du sujet, de ses désirs et de ses forces.

9 Il importe donc de ne pas oublier que la stratégie mélancolique, qui peut s'avérer nécessaire pour la survie psychique, n'en reste pas moins une adhésion à des fausses images du Bien : des fétiches, s'interposant entre les hommes et la vérité - une situation que les Docteurs de l'Église appelaient recessus a bono divino, la « fuite de l'homme devant la richesse de ses possibilités spirituelles »:

Que l'acidiosus s'écarte de sa fin divine ne signifie nullement qu'il parvienne à

l'oublier ou qu'il cesse, en réalité, de la désirer (...). Le recessus traduit moins une

éclipse du désir que la mise hors d'atteinte de son objet : il s'agit d'une perversion de la 
volonté qui veut l'objet, mais non al voie qui y conduit, et qui tout à la fois désire et barre la route à son propre désir (...). Dans la Summa theologica, l'acedia n'est pas opposée à la sollicitudo, c'est-à-dire au désir et à l'intérêt, mais au gaudium, c'est-à-dire à l'assouvissement de l'esprit en Dieu'. Rancière incarnent moins une tentative de présentifier l'absent qu'une manière pour laisser l'absent dans son absence tout en continuant à désirer sa présence. Une manière, certes, de ne pas céder sur son désir, mais qui n'est pas exempte d'impasses potentiellement désastreuses.

\section{III} pourra suggérer que de nombreuses pratiques de l'activisme et de l'engagement contemporains veulent en quelque sorte l'objet, mais ne peuvent, ou, parfois, ne veulent plus accéder aux moyens qui permettent de l'atteindre. Leur « mélancolie » est donc une impasse de la réalisation: c'est pourquoi leurs formes dominantes se présentent, selon l'expression de J. Rancière, comme des "mises en scène" censées produire un "bonheur» immanent, intransitif, autosuffisant et autophage, donc à l'abri de toute vérification par un rapport à l'altérité traumatique du réel.

Dans quelle mesure est-il possible de transposer ces analyses à d'autres phénomènes que les mouvements mentionnés par Rancière? Il convient peut-être de creuser davantage cette référence à la «mise en scène » qui s'est glissée sous la plume du philosophe.

Une mise en scène est un acte qui reste en deçà de la réalité, qui ne sort pas des limites de la fiction et de la simulation maitrisées, qui ne transforme pas le monde au-delà de la sphère limitée de sa construction. Mais elle est aussi une pratique artistique, qui s'inscrit dans des coordonnées esthétiques et culturelles déterminées. Une mise en scène n'est pas une simple fiction : c'est une fiction qui produit une «belle apparence» (le Schein de la théorie esthétique postkantienne), un geste qui a tendanciellement la consistance des œuvres de l'art et de la pensée.

Or les formes, les concepts, le savoir, les objectivations de l'« esprit » et les pratiques de la " culture » confèrent une certaine maitrise au sujet qui se soumet à leurs normes ; mais une telle maitrise ne sort pas de l'espace séparé, autoréférentiel et immanent, que de telles normes instituent et distinguent comme la condition de leur validité. D'où l'effet de déplacement, de compensation, et finalement de "consolation " aliénée que ces formes peuvent exercer. C'est ce que Marcuse appelle la « fonction affirmative de la culture » :

La culture affirmative a adopté, avec l'idée de la pure humanité (Humanität), la revendication historique du bonheur universel des individus (...). Le niveau le plus élevé qui puisse être atteint par l'homme suppose la réalisation d'une communauté de personnes libres et raisonnables dans laquelle chacun a la même possibilité de développer et d'épanouir ses possibilités (...). L'unité représentée par l'art, la pure humanité de ses personnages est irréelle; elle est l'image inversée de ce qui se produit dans la réalité sociale ${ }^{10}$.

Les "mises en scène" dont parle Jacques Rancière relèvent d'une telle fonction affirmative : elles déplacent dans un espace fictif et séparé des désirs et des capacités qui ne peuvent s'inscrire dans la transformation effective de la réalité sociale. 
14

Notre hypothèse est que cette "fonction affirmative" ne se limite pas aux grands rassemblements et aux occupations des places mentionnés par J. Rancière. Il y a une tendance générale, qui affecte la plupart des formes contemporaines du militantisme, de l'activisme ou de l'engagement, à faire des pratiques et des processus politiques des actes et des objets esthétiques et/ou culturels.

Ce glissement de la politique vers la culture et l'art n'est pas une nouveauté absolue : du Surréalisme de l'entre deux guerres au Situationnisme, jusqu'aux nombreux programmes des années 1970 visant à « dynamiter le langage » et à assimiler la révolution au langage poétique, tout un pan des séquences politiques au XXème siècle à essayé de faire coïncider transformation du monde, changement de la vie et modification des formes habituelles de l'activité artistique et intellectuelle.

Ces opérations ont généralement abouti à confondre les plans qu'elles étaient censées unifier, et ont fini par concentrer tous les efforts réels dans la sphère de la culture, la plus immédiatement accessible pour des intellectuels. Le discours sur la Révolution se voulant immédiatement une révolutionnarisation du Discours, et vice versa, l'activité discursive finissait par se croire capable de casser en deux l'histoire du monde et de transfigurer l'existence de tous et de chacun.

Mais, à côté de ces tentatives pour annuler toute médiation entre politique, culture et vie quotidienne, il continuait d'exister des sites politiques dont l'autonomie et la logique propres étaient incontestables - le mouvement ouvrier, la Troisième Internationale, les luttes anti-impérialistes... - et dont la consistance permettait et imposait aux gestes et aux produits de l'Esprit de vérifier la tenue de leurs orientations et de leurs effets. Ce n'est qu'à partir de la fin des années 1970 et de la dissolution à la fois de la centralité ouvrière et de la perspective communiste, que ces sphères irréductibles et «objectives » ont cessé de constituer la mesure et l'horizon des comportements militants.

Depuis la fin de la dernière séquence rouge du XXème siècle, ces comportements ont changé de statut: ils ont commencé à viser moins la transformation des conditions générales (économiques, sociales, institutionnelles...) de la vie de chacun que le simple témoignage de l'existence d'un secteur de l'opinion, d'une minorité ou d'une sensibilité ou alors la constitution de communautés restreintes cimentées par un vocabulaire et des symboles exclusifs et de plus en plus autoréférentiels.

Ainsi, le « militant » tend de plus en plus à devenir un professionnel de la « visibilisation » d'un point de vue, d'une « cause » ou d'une série de valeurs, et de la cohésion/animation de groupes réunis autour de certains intérêts, traditions ou drapeaux Ce qui veut dire que le militantisme «milite » de moins en moins, du moins au sens que ce mot pouvait avoir entre 1917 et 1977, et se réduit progressivement à déclarer des désirs ou à consolider des identités.

17 C'est précisément cette configuration qui impose en quelque sorte le glissement de la pratique politique, indissociable de sa vérification par l'altérité, vers la maitrise et l'efficacité purement intransitives des pratiques de la culture.

\section{IV}

18 Au XXème siècle, en tout cas après Que faire? de Lénine (1902), un militant est un professionnel. Cela ne veut pas dire qu'il reçoit un salaire, comme un permanent, mais 
qu'il applique systématiquement ses efforts en vue de l'obtention d'un résultat. Dans les Carnets de prison, Gramsci a développé les implications de cette idée :

L'homme politique authentique a l'intuition simultanée de l'idée et de son processus réel d'actualisation : il esquisse à la fois le projet et le "protocole » pour sa mise en exécution (...) Le projet doit être saisi par tous les acteurs, de telle sorte que chacun voie clairement la tâche qui lui revient dans sa réalisation (...) et que, lorsque le projet suggère une action, il en fasse voir en même temps les conséquences négatives et positives (...) tout en fournissant un fondement organisationnel ${ }^{11}$.

Le militant politique est par conséquent une nouvelle figure de la fonction intellectuelle, un spécialiste de la réalisation méthodique des « idées »:

Tout grand homme politique ne peut ne pas être un grand administrateur (...), un grand organisateur. Cela peut constituer un critère de jugement : le théoricien, le concepteur de plans, est évalué en fonction de ses qualités d'administrateur, là où " administrer » veut dire prévoir les actes et les opérations - depuis leur niveau «moléculaire » jusqu'aux actions les plus complexes - nécessaires pour réaliser le plan $^{12}$.

Quelques années avant l'emprisonnement de Gramsci, à l'époque où celui-ci animait la tentative révolutionnaire italienne qui aurait scellé sa destinée, un autre analyste des conditions modernes de la politique, à savoir Max Weber, mettait en évidence le rôle grandissant de la formation spécialisée dans la vie politique et sociale moderne :

Le développement de la technique de la guerre a fait naitre l'officier professionnel, l'affinement de la procédure juridique a produit le juriste doté d'une formation spécifique. Sur ces terrains, le fonctionnariat spécialisé l'a définitivement emporté, dans les États les plus développés, au XVIème siècle ${ }^{13}$.

Les fonctionnaires modernes sont « une couche de travailleurs intellectuels dotés d'une compétence professionnelle spécialisée, grâce à une formation qui s'étend sur de longues années ${ }^{14}$; le «révolutionnaire professionnel » appelé par Lénine de ses vœux, et tendanciellement réalisé dans le modèle bolchevik du militant, constitue la tentative de faire coïncider ces compétences spécialisées avec la subjectivité du "chef politique » responsable devant une cause, dont l'honneur «consiste précisément à assumer la responsabilité personnelle exclusive pour ce qu'il fait $»^{15}$.

Ce type spécifique qu'est le militant moderne est donc soumis en permanence à une vérification pratique de son engagement, à travers, d'une part, les savoirs et les compétences auxquels il doit soumettre sa conduite, et, d'autre part, par la responsabilité à l'égard de la cause qu'il sert - une responsabilité qui n'est pas purement subjective, car elle relève de l'inscription de cette cause dans le monde réel, donc de la réalisation d'une idée politique, et doit ainsi « assumer les conséquences (prévisibles) de son action ${ }^{16}$.

21 Autrement dit, selon Gramsci et Weber, le militant "moderne » doit sans cesse rechercher et vérifier les moyens adéquats pour rendre efficace sa conduite et pour augmenter les chances de réaliser son projet : un tel militant est un spécialiste du rapport entre moyens et fins, et il n'a pas le droit de se détourner de cet horizon de réalisation possible pour se limiter à énoncer les valeurs dont il s'inspire ou les désirs qui le motivent.

On s'est peut-être excessivement habitués à l'idée que les grands moments de la politique consistent en de pures "prises de parole", et que toute rationalisation des conduites dans cette sphère ne serait que de l'expropriation bureaucratique de la créativité collective. La parole libérée peut tout aussi bien constituer un moyen pour combler dans l'imaginaire des lacunes réelles, et il n'y a rien de moins créateur que des mots sans 
conséquences. C'est du moins la position de Jules Andrieu, un rescapé de la Commune de Paris qui esquisse un bilan très critique de cet épisode tant admiré par les théories radicales contemporaines :

Une grande faute a été commise par la Commune tout entière : celle d'avoir copié toutes les autres assemblées et d'être descendue par la pente fatale des choses au rôle stérile d'un parlement. Là où il y a des bureaux constitués en vue de l'exécution d'une besogne taillée à l'avance, les beaux discours ou les grands parleurs ne trouvent plus l'emploi de leurs facultés; il y a seulement place pour les capacités, pour le dévouement et pour l'assiduité au travail. Mais si vous donnez une situation difficile à liquider à soixante-dix ou quatre-vingts hommes réunis en mode parlementaire, l'illusion et la tendance à abuser du pouvoir ne tarderont pas à créer une majoritét ${ }^{17}$.

Un texte prophétique, qui annonce certes Lénine et Gramsci, mais aussi les impasses contemporaines et la substitution de la parole à la praxis.

\section{V}

Une anecdote. Dans un café, vers la fin des mobilisations contre la loi ORE et Parcoursup du printemps 2018. Au cours d'une conversation, je qualifie de "spectacle» les actions menées pendant les manifestations par les soi-disant militants black bloc (qui sont rapidement devenus l'aspect le plus médiatiquement visible de cette vague de protestations). Des étudiants assis peu loin entendent le mot; ils ont participé aux manifestations et aux blocages, veulent comprendre pourquoi et en quel sens je parle de « spectacle».

En racontant leur expérience des manifestations, ils disent trouver dans la chorégraphie des actions susmentionnées quelque chose qui protège - symboliquement, sans doute ${ }^{18}$ de la violence disproportionnée employée par la police, une manière de transformer la peur en autre chose. Ils appellent cela « une expérience cathartique ».

L'adjectif «cathartique » reste une expression très révélatrice, puisque la catharsis - au sens de la libération d'affects qui n'arrivent pas à déboucher autrement - est précisément l'effet produit par la représentation fictive d'une action, du moins selon la première théorie du spectacle en Occident, la Poétique d'Aristote.

Le récit des étudiants énonçait ainsi une vérité profonde : la satisfaction que peuvent fournir certaines pratiques actuelles vient du traumatisme, de la peur et de la douleur, qu'elles essayent de mettre à distance. En ce sens précis, parler de «catharsis » est plus pertinent que toute affabulation sur l'«ambiance» des AG ou sur la "puissance collective " des manifestations - des mots qui ont effectivement été employés par des participants aux mobilisations récentes (et moins récentes) : ces étudiants donnaient voix à une négativité que la rhétorique des « passions joyeuses » dominant la discursivité de la gauche radicale est incapable de reconnaître et de formuler.

Cette «catharsis » renvoie ainsi à un travail sur les émotions à partir d'un état négatif, d'un blocage ou d'une impasse, et pourrait être interprétée à travers l'analyse proposée par Sartre, qui part précisément d'une situation d'impasse insurmontable sur le plan de la praxis :

Une émotion (...) c'est une transformation du monde. Lorsque les chemins tracés deviennent trop difficiles ou lorsque nous ne voyons pas de chemin, nous ne pouvons plus demeurer dans un monde si urgent et difficile. Toutes les voies sont barrées, il faut pourtant agir. Alors nous essayons de changer le monde, c'est-à-dire 
de le vivre comme si les rapports des choses à leurs potentialités n'étaient pas réglés par des processus déterministes ${ }^{19}$. au sujet ne peut faire oublier l'ineffectivité réelle de ses opérations :

La conduite émotive n'est pas sur le même plan que les autres conduites, elle n'est pas effective. Elle n'a pas pour fin d'agir réellement sur l'objet en tant que tel (...). Elle cherche à conférer à l'objet par elle-même, et sans le modifier dans sa structure réelle, une autre qualité, une moindre existence, ou une moindre présence (...). Si l'émotion est un jeu, c'est un jeu auquel nous croyons ${ }^{20}$.

\section{VI}

La spectacularisation des manifestations, leur valeur émotionnelle et esthétique remplaçant leur inscription possible dans un processus rationnel et effectif, ne sont pas des phénomènes récents. Un texte de Franco Fortini les évoquait déjà il y a presque quarante ans. Ce texte vise un projet de meeting à Bologne, en 1981, sollicité entre autres par Il Manifesto, où des intellectuels et des dirigeants politiques de gauche expliqueraient aux «jeunes » ce qui s'est (réellement) passé en Italie entre 1969 et 1981 - les grèves de l'automne chaud, les attentats à la bombe, le terrorisme, les tentatives de coup d'État, l'involution autoritaire et conformiste des années de l'état d'urgence et de la «solidarité nationale » entre les forces parlementaires... Selon Fortini, une telle initiative «ne pourra pas ne pas être un spectacle ${ }^{21}$, car elle ne pourra pas proposer aux jeunes conviés « un organigramme des réalités internationales et nationales, un tableau vraisemblable de la société et de son mouvement, un catalogue d'ennemis, d'adversaires, d'amis et d'alliés (...): bref, un programme politique ", ni impliquer des conséquences réelles concernant la classe politique italienne et les rapports de pouvoir en place ${ }^{22}$.

Fortini caractérise parfaitement le modèle d'intervention politique qui est devenu dominant dans toutes les variantes de ce que fut la " gauche » : des actes qui n'ont aucune conséquence, qui ne produisent aucun effet au-delà d'eux-mêmes et de leur simple présence, et dont le seul but est de susciter des affects "positifs", rassurants et protecteurs, chez les participants, du fait de se trouver partager des mots, des gestes, des symboles, des jugements, et évidemment des inquiétudes, des frustrations et des peurs.

Quarante ans plus tard, de tels actes ont fini par constituer l'essentiel de ce que «politique» veut dire, du moins si l'on n'entend pas par ce mot les élections, le storytelling pré- et post-électoral, la gestion des affaires courantes ou la petite chronique des décisions et des indécisions des élus. En 1981, la réduction des actes politiques à des gestes spectaculaires était la conséquence d'un «traumatisme historique, italien et mondial, trop important », qui rendait «trop abyssal le processus de récupération de la parole et de l'action $»^{23}$.

Depuis cette époque-là, la parole et l'action n'ont eu de cesse de se dérober. Les valeurs «spectaculaires » affectent depuis longtemps les pratiques les plus traditionnellement liées au mouvement ouvrier et aux paradigmes politiques du XXème siècle. Depuis des décennies, les défilés, les rassemblements et les grèves visent davantage à attester l'existence des organisations politiques et syndicales qui les promeuvent, ou de quelques catégories socio-professionnelles porteuses de revendications spécifiques, qu'à modifier les rapports de force politiques suivant un projet d'hégémonie, à orienter la politique 
économique et l'organisation du travail, ou à affirmer un pouvoir réel sur les lieux de la production et dans l'espace urbain.

Les manifestations publiques et les actions collectives ne gardent plus aucun lien avec ces visées, dont le contenu effectif est d'ailleurs devenu totalement opaque depuis la fin des années 1970, jusqu'à ce qu'elles disparaissent des consciences. Des pratiques restent, mais comme des purs rituels, des simulacres dont la répétition rassure en quelque sorte mais qui ne renvoient plus à des «schèmes de coordination entre l'interprétation des situations, la détermination des actions et l'entretien des affects $»^{24}$, qui ne s'articulent plus à des procédures de vérification politique susceptibles de les interroger et le cas échéant de les modifier.

\section{VII}

On peut s'étonner de la rareté des études consacrées aux formes actuelles de militantisme; mais encore plus rares sont les espaces de confrontation où des individus engagés dans des activités ou des mouvances politiques pourraient discuter de leurs projets, mobiles, orientations et impasses au-delà de la simple récitation des tics de tel ou tel groupe, de tel ou tel Maître à penser.

Tout se passe comme si les «militants » actuels, les animateurs de ce que Rancière appelle les « oasis » et Enrico Donaggio les « lieux communs d'humanité » ${ }^{25}$ - l'auteur italien est d'ailleurs bien plus conscient de la nature pré-politique de ces formes -, disposaient de paroles et de discours suffisants pour affirmer leur existence, pour tenir en tant que membres de collectifs plus ou moins organisés, mais restaient largement incapables de problématiser devant autrui, et donc de soumettre à l'épreuve de la Verfremdung, leurs principes et leurs pratiques. Il n'existe aujourd'hui aucune sphère publique critique où ces pratiques pourraient être mises en partage par-delà le cercle restreint de leurs porteurs immédiats.

C'est pourquoi il arrive que la condition actuelle du militantisme fasse plus facilement l'objet de portraits littéraires que d'essais de théorie critique. C'est le cas d'un livre récent de Nathalie Quintane, qui esquisse un parallélisme instructif entre deux figures contemporaines de l'engagement : le " bénévole » et le " militant anti-répression », celuici étant un autre nom des black bloc ou du « cortège de tête ».

Les anti-rép. sont des artistes; des artistes dramatiques par exemple. Ils ne sont pas vêtus en tous les jours, ou leur mise est soignée, calculée : le noir et sa symbolique dominent; c'est aussi une couleur qui affine, mincit, rend élégant. Je suis le ténébreux, le veuf, linconsolé. La capuche qui couvre leur tête et dissimule leur visage leur donne du mystère (...). Les gestes qu'ils font appartiennent à une chorégraphie répertoriée, photographiée, archivée, montrée dans les musées (...). Ils ont quelques minutes pour la rendre vivante, pour qu'on y croie ${ }^{26}$.

Les « bénévoles ", eux, ne sont pas des artistes mais des " travailleurs » :

Compartimentés par l'État et les associations qui ont délégation, c'est-à-dire qui font ce que l'État ne veut plus faire, ils lâchent toute perspective de sortie du compartiment, toute prétention aux allers et retours, pour s'offrir à la tâche qu'on a choisie pour eux ou qu'ils se sont choisie - ils font don de leur personne. On distribue la bouffe, on trie les vêtements, on véhicule, on remplit les papiers, on explique, on héberge ; que voulez qu'on fasse de plus ?27

Ces lignes de N. Quintane montrent très bien la nature " artiste » des comportements de ceux qu'elle appelle les anti-rép. Mais la symétrie avec les bénévoles est peut-être poussée 
trop loin. Car elle suggère que les bénévoles ne seraient que des purs corps compatissants, faisant des gestes élémentaires dépourvus de statut symbolique.

En réalité, les «bénévoles » et leurs associations consacrent beaucoup de leur temps à organiser des marches ou des meetings, par exemple pour commémorer les migrants noyés dans la Méditerranée; ils écrivent des lettres ouvertes à la presse, aux institutions et aux élus, ils construisent des initiatives publiques (tel les «États Généraux de la Migration »), ils participent à des rencontres avec des députés, des dirigeants des partis, des syndicalistes, d'autres associations...

C'est une partie très importante du travail du bénévole, et elle semble très souvent ne déboucher que sur les mêmes effets « cathartiques » que produit la chorégraphie lugubre du cortège de tête: c'est des actes que l'on accomplit pour tenir, pour affirmer sa présence, son être-au-monde, et pour reconstituer un espace minimal de maîtrise sur ses actes et ses paroles. Sinon, l'impuissance pourrait s'avérer écrasante, puisque les bénévoles sont exposés à un réel qu'ils reçoivent souvent en pleine figure, et qui n'est pas moins violent qu'une charge de police: non seulement à cause de la rencontre traumatique avec des individus marqués par une très grande détresse, mais aussi à cause de la contradiction mortelle qui consiste à lutter contre la volonté politique d'institutions dont les bénévoles assurent en même temps une partie grandissante des missions... ${ }^{28}$.

Ainsi, les bénévoles aussi font de la "culture ", assurent des "mises en scène », et les activités par lesquelles ils déclarent leur être-là sont souvent incapables de modifier les décisions étatiques qui font des migrants une population grandissante d'individus précarisés et hors-la-loi, tout comme une place occupée ne peut rien contre le capitalisme financier ou les politiques d'austérité, ni un ballet de guérilleros urbains contre les dérives autoritaires des États et la montée en puissance de l'extrême droite.

Finalement, il serait injuste et myope de sous-estimer, parmi les pratiques des bénévoles, précisément leurs gestes élémentaires, qui sont au moins obligés de s'articuler à un désir autre que celui des bénévoles eux-mêmes - du moins virtuellement, car la subjectivité militante contemporaine est souvent trop fragile pour renoncer facilement à jouir dans/ de l'immanence de ses propres pratiques, si bien que la tentation potentiellement mortelle des bénévoles ne consiste pas à s'arcbouter excessivement sur les «choses à faire », dans leur difficulté ordinaire - démarches auprès de la préfecture, recherche de logements et de nourriture, etc. -, mais précisément à exorciser leur impuissance à gérer ces tâches en déplaçant leurs efforts vers des activités plus épanouissantes (des ateliers d'écriture poétique ou de photographie, du théâtre et de la musique, etc., voire des conférences de presse et des interventions dans les médias).

Cependant, il y a encore, dans les pratiques apparemment les plus anonymes, la trace de ce que Brecht appelait la Weltfreundlichkeit :

Dans les Sermons domestiques, Brecht a écrit une ballade sur les gentillesses de ce monde. Il y a en a trois : la mère nous lange, le père nous tend une main, des gens répandent de la terre sur la tombe (...). Les témoignages de la gentillesse du monde nous sont apportées aux moments les plus durs de l'existence : à la naissance, lors du premier pas dans la vie, au moment de la quitter. Voilà le programme minimum de la sollicitude humaine ${ }^{29}$.

Jean Oury aussi a résumé la teneur éthique de l'expérience de La Borde par ce mot de "gentillesse ", employé par la mère de l'une de ses patientes :

Ça tourne là autour d'un tenir à tout prix. Les gens qui se présentent là, coûte que coûte il ne faut pas les laisser tomber. C'est ça qui résume la trame de base quelqu'un qui vient, on ne peut pas le laisser tomber (...). Pas de la sympathie, de la 
gentillesse. Même si on gueule comme des ânes, cette gentillesse, c'est de ne pas laisser tomber ${ }^{30}$.

Loin de se réduire à des ouvriers-masses de la solidarité, les "bénévoles " gardent un rapport à des gestes qui peuvent donner accès, du moins virtuellement, à cette dimension où quelque chose se fait jour par-delà le « spectacle ».

\section{VIII}

5 On dira que ces descriptions sont partielles, que ce tableau de la situation actuelle est unilatéralement sombre, que ce portrait du militantisme contemporain est uniquement à charge et qu'il néglige ou défigure tout ce qui est fait, envers et contre tout, et au prix d'efforts parfois épouvantables, dans les innombrables « lieux communs d'humanité » qui résistent malgré tout.

En réalité, ces tentatives d'analyse - sans doute partielles et impressionnistes, mais il est difficile de dire comment on pourrait les rendre plus complètes, rigoureuses et «scientifiques » - n'ont de sens que si elles peuvent contribuer à saisir la valeur et la consistance de « ce qui est fait » en dépit de tous les obstacles et les difficultés. Mais, pour ce faire, il est indispensable de ne pas s'aveugler sur la situation générale à laquelle quelques résultats sont difficilement arrachés.

37 La situation, généralement parlant, est loin d'être bonne. Après la crise financière de 2008, il était possible d'imaginer un renversement ou du moins un changement dans la conjoncture en Europe et dans le monde : la Grèce de Syriza incarnait l'espoir de rejouer les rapports de force à l'échelle européenne et de s'opposer d'un point de vue socialiste à la politique de l'UE; à la même époque, les révoltes dans les pays arabes montraient qu'il est possible de renverser des régimes autoritaires et les expériences socialistes en Amérique Latine indiquaient la possibilité de donner vie à des formes nouvelles, égalitaires, d'organisation politique et économique. Le capital, l'État et l'impérialisme semblaient redevenir des réalités visibles et contingentes, donc modifiables.

De tous ces possibles, il ne reste aujourd'hui pratiquement rien. Les mobilisations anticapitalistes, de 2008 à 2016, ont été incapables, non seulement d'ébranler les rapports capitalistes de production, mais aussi, plus modestement, d'imposer des limites aux politiques d'austérité, à la destruction des services publics et à la dégradation du travail salarié. Le monde est de plus en plus secoué par des conflits inter-impérialistes ; partout les États évoluent dans un sens de plus en plus policier et autoritaire, qui affecte désormais jusqu'aux piliers libéraux classiques, tels l'équilibre des pouvoirs, l'indépendance de la magistrature ou la liberté de la presse ; l'Union Européenne a peutêtre perdu toute capacité d'hégémonie politique, mais elle se contente d'assurer la reproduction du capital et le pouvoir de ses fractions dominantes au sein de son espace institutionnelle.

De plus, les mots d'ordre et certains des affects des mobilisations qui ont suivi la crise financière ont été rapidement récupérés et absorbés par la montée en puissance d'une sorte d'extrême droite post-moderne, violemment europhobe mais aussi entièrement homogène au capitalisme le plus sauvage, et que les dirigeants libéraux de l'Europe ont sciemment ménagée au moment même où ils refusaient toute mise en question de l'ordre économique établi. Ainsi, le mot d'ordre des « peuples contre les élites », et des signifiants 
tels que « peuple » ou " populaire », ont glissé sans difficulté du discours de la solidarité à l'égard des salariés grecs aux aboiements racistes du ministre italien de l'intérieur.

39 A la fin de cette séquence initiée entre 2007 et 2008, non seulement l'état des rapports de force, mais aussi les dispositions subjectives de vastes populations, autorisent toute forme de pessimisme. Le cas italien est, encore une fois, un laboratoire extrêmement instructif : les gouvernants actuels bénéficient d'un consensus de masse que les mesures les plus brutales à l'égard des migrants ne font que renforcer, et ils ont réussi, trois mois après la formation du gouvernement, à s'imposer dans les anciennes forteresses du Parti communiste, de la gauche et du syndicalisme de lutte.

Le sociologue et historien Marco Revelli a résumé efficacement la situation :

Les gens savent très bien pour qui ils votent. Ils savaient qu'ils étaient en train d'approuver la «méchanceté » de M. Salvini [ministre de l'intérieur et chef de la Ligue, A.C.], sa politique de la cruauté. Ils savaient qu'ils étaient en train de voter pour ceux qui ont déclaré la guerre aux bateau des ONG qui sauvent les migrants dans la Méditerranée, pour ceux qui préconisent leur mise au ban ou qui voudraient, le cas échéant, les faire couler ${ }^{31}$.

40 Une telle situation est donc définie non seulement par l'extrême disproportion des forces qui rend l'échelle des structures et des stratégies entièrement inatteignable pour les pratiques militantes «locales", mais aussi par le fait incontournable d'une adhésion de plus en plus massive à des discours et à des conduites qui rendent les hommes plus inégaux et plus vils, et le monde plus sombre.

Parmi les impasses actuelles, celle-ci est peut-être la plus sidérante, la moins facilement tolérable pour la conscience des militants des «bonnes pratiques»: le fait que «les hommes aiment mieux les ténèbres que la lumière », comme le dit l'Evangile de Jean, qu'ils choisissent de devenir des bourreaux volontaires, non pas au nom de la peur, d'une colère mal dirigée, ou d'intérêts pragmatiques calculables, mais uniquement de la jouissance obscure d'humilier et de mépriser ceux qui sont déjà humiliés et méprisés.

41 Cette séparation des êtres, cette distance infranchissable entre les consciences, qui fait que certains haïssent et veulent détruire les gestes et les conduites que d'autres aiment et entretiennent, tous les signes de l'existence réelle de l'Ennemi, sont un scandale devant lequel il est facile de détourner le regard: il constitue un défi non seulement pour notre capacité de comprendre et de vouloir comprendre ce que pensent réellement nos semblables, mais aussi pour l'anthropologie spontanée de la subjectivité " progressiste ", qui postule chez l'homme une tendance vers le Bien et le Vrai dont les déviations ne seraient que des erreurs ou des quid pro quo.

\section{IX}

Il serait peut-être inutile de rappeler tout cela, s'il n'était pas évident que, dans de nombreux discours plus ou moins savants, voulant accompagner, interpréter, voire, malgré toutes les dénégations, diriger les mobilisations et les pratiques engagées, la réalité de cette situation est radicalement exorcisée.

43 Jacques Rancière le constate : dans une certaine discursivité « radicale ", les défaites, les impasses et les insuffisances sont occultées par la conviction que, quoi qu'il arrive, une logique sous-jacente aux processus sociaux garantit la continuité et la validité des mots d'ordre, des conceptualisations et des langages : 
Il faut sortir de cette logique qui enrôle l'évolution historique au service de ses désirs, en interprétant l'histoire de la domination comme celle d'un monde d'apparences voué à se dissiper au profit de la réalité nue ${ }^{32}$.

Les cibles polémiques de J. Rancière sont nombreuses : le «Comité invisible », qui épouse la « vision heideggerienne d'un monde décadent, appelant un retournement radical $»^{33}$; les discours dominants su le « néolibéralisme » qui y voient « le moment où la domination économique se montre a nu dans la dissolution de toutes les croyances et institutions ${ }^{34}$; les théoriciens qui voient dans les transformations du capitalisme "le signe que c'est désormais la vie entière et non plus la force de travail qui est requise par le capitalisme postfordiste et en déduisent l'émergence d'un mouvement biopolitique, un mouvement de la vie elle-même succédant au classique mouvement ouvrier $"{ }_{. . .35}{ }^{35}$

Tous ces discours fournissent une lecture "globale" de la phase présente, et la présentent comme globalement marquée par des "avancées ", par ce qu'Éric Hazan appelle «un grand changement subjectif dans les façons de lutter contre l'ordre existant $»^{36}$. Mais ce sentiment de continuité, d'évidence, presque de facilité, se dégage aussi de formules telles que la « convergence des luttes » ou la « riposte populaire », qui présupposent évidemment que des «luttes» existent ayant un statut univoque et une cohérence au moins virtuelle, qu'un " peuple » soit donné en tant que sujet politique, et que « peuple » soit un nom adéquat pour un tel sujet politique...

C'est des discours, ou des fragments de discours, qui visent à totaliser la situation présente et à en dégager une tendance vers un dénouement - une tendance par rapport à laquelle les impasses, les échecs, la pure et simple fatigue que rencontre la volonté de continuer, malgré tout, à tisser des « lieux communs d'humanité », apparaissent comme des simples accidents: des prises de conscience insuffisantes ou des erreurs tactiques. Tout point critique, toute difficulté pouvant révéler le signe d'une contradiction sont ainsi réduits à un rien ontologique, à la simple privation accidentelle d'une plénitude toujours-déjà garantie.

Selon J. Rancière, ce schéma ne fait que répéter l'ancienne (et illusoire) idée marxiste que la révolution ne ferait que libérer des «potentialités déjà formées par la marche du monde en avant ${ }^{37}$. On peut indiquer d'autres raisons de s'en méfier. De tels discours constituent, encore une fois, un écran qui empêche de penser ce qui se passe réellement dans les pratiques militantes contemporaines, ce qui s'y fait et surtout ce qui ne s'y fait pas. Les vastes édifices discursifs qui composent le "sens commun », ou la " philosophie spontanée ", d'importants secteurs "radicaux " contemporains fonctionnent moins comme des analyses ou des propositions stratégiques que comme des signes d'identification de courants, groupes et tendances: ils produisent un effet de reconnaissance qui permet à des milieux et à des communautés de garder une certaine cohésion indépendamment de l'échec ou du succès de leurs « lignes ».

Mais les édifices que vise Jacques Rancière indiquent un autre phénomène, irréductible aux emplois directement liés aux jargons et aux chapelles. Car de tels discours fonctionnent en outre au sein d'une discursivité plus générale et diffuse, aux contours mal définis, qui produit des schémas d'interprétation et d'expression anonymes et irréfléchis. 


\section{$\mathrm{X}$} essentiellement de morceaux et de débris du marxisme, de la phénoménologie, de la psychanalyse $\Perp^{38}$ : une métaphysique dont la fonction consiste à instituer des évidences et des clartés là où il y a de l'obscurité et de l'inintelligible, des réflexes et des réponses immédiats là où il y aurait matière à réfléchir et à rester perplexe. Il me semble qu'une telle métaphysique commune consiste aujourd'hui, pour des vastes strates d'intellectuelsmasses - étudiants, enseignants, chercheurs, écrivains divers, artistes, «intermittants ", activistes d'associations et de groupes, etc. -, dans des « morceaux et débris » empruntés à Foucault, Deleuze, Badiou, Negri, Rancière, Agamben, Žižek, le Comité Invisible, Butler..., sans oublier évidemment Marx, l'École de Francfort, Althusser (moins fréquemment), David Harvey, désormais Tronti, et les paradigmes collectifs (French Theory , Italian Theory, Gender Studies, Subaltern Studies, Postcolonial Studies...)

C'est une discursivité "radicale» qui articule transversalement discours militants, initiatives éditoriales grandes et petites, colloques et séminaires universitaires, « grands noms » de la pensée et travailleurs intellectuels subalternes, et qui est effectivement commune à des secteurs assez nombreux et variés de la «scène » intellectuelle contemporaine: un peu comme, entre les années 1950 et 1960, en France, s'était constituée une "métaphysique commune » dont les évidences reposaient sur un mélange d'existentialisme, hégélianisme (phénoménologique), marxisme (daté 1844) et psychanalyse (à la sauce herméneutique), avant d'être recouverte par une autre métaphysique - que Jean-Claude Milner appelle la «doxa structuraliste» - et par ses évidences propres (le signifiant, la coupure épistémologique, la « mort de l'homme »...).

Une certaine discursivité actuelle n'opère pas très différemment avec des formules telles que « partage du sensible », « pouvoir constituant» (ou « destituant»), «formes de vie », "guerre civile", «émancipation", «événement ", «tumultes ", ou plus classiquement « révolution », « communisme », « Commune » (de 1871), voire " peuple » ou « luttes »...

Faut-il le préciser? Le texte que le lecteur a devant les yeux, son auteur, la publication dans laquelle il parait, et sans doute beaucoup de ses lecteurs, participent de cette discursivité, la reproduisent à travers telle ou telle référence, tel ou tel système d'associations et d'allusions s'établissant entre des noms, des concepts et des vocabulaires.

Cest pourquoi il ne saurait être question de disqualifier cette discursivité pout lui opposer d'autres évidences, d'autant plus que ce réseau de discours englobe des contenus très disparates et inégaux, dont chacun mériterait, pris en tant que singularité, une analyse spécifique - mais c'est justement leur fonctionnement dans un dispositif qui efface les singularités, les aspérités et les contradictions qui nous intéresse ici.

La discursivité «radicale» dont il est question fonctionne, dans ses aspects d'homogénéité massive, en produisant des identités, des continuités et des reconnaissances : son effet consiste à absorber le réel des pratiques, leur contingence et leurs blocages douloureux, dans la simplicité d'un principe d'explication que la linéarité d'un discours permet de restituer en transparence. Autrement dit, c'est la tendance à déduire les pratiques politiques de la Philosophie politique, et à effacer par là de ces 
pratiques tous les aspects traumatiques et dérangeants, les questions possibles à propos de ce qui marche et de ce qui ne marche pas, et des raisons des succès et des échecs.

La persistance et la multiplication de telles discursivités empêchent précisément de reconnaître que dans les pratiques militantes actuelles (et, par effet de rétrospection, dans celles du passé récent), il y a quelque chose d'opaque, qui échappe aux savoirs disponibles : qu'est-ce qui fait tenir une pratique, un groupe, un engagement? Qu'est-ce qui se passe quand cela, au contraire, ne tient plus? Qu'est-ce qui déclenche un processus politique réel? Quel est le ressort subjectif de la recherche d'une vérification par la pratique? D'où vient, et comment soutenir, le désir de ne pas s'empêtrer dans la mise en scène de soi-même et de se confronter à l'altérité traumatique des situations effectives, de leurs lacunes et de leurs béances?

Sans oublier toutes les questions particulières qui se posent au niveau de la singularité des pratiques mais qui finissent toujours par l'excéder: comment éviter qu'une revendication socio-professionnelle reste purement corporative ? Comment faire en sorte qu'une expérience d'autogestion ne dérive vers une utopie isolationniste? Comment organiser dans la durée l'accueil des personnes migrantes? Comment se positionner face aux conditions de leur régularisation imposées par l'État, mais aussi aux demandes venant de ces personnes? Comment enraciner une pratique dans un territoire sans l'exclure d'un horizon plus vaste? Comment une grève ou un blocage peuvent réellement influencer une négociation ou un projet de loi?

Et encore : comment inscrire des bonnes pratiques dans la position que chacun occupe au sein de professions et d'institutions de plus en plus normalisées et surveillées? Comment concilier l'engagement avec la précarité grandissante des situations matérielles ? Peut-on encore faire un bon usage du travail social, de l'éducation, de la recherche, de la médecine - ou du syndicalisme ? À quelles conditions ? Et, là où ces conditions ne sont pas remplies, comment éviter que la prise de distance éventuellement nécessaire ne se transforme en repli ou en sécession silencieuse? Comment organiser et rationaliser les refus et les adhésions?

Pour chacune de ces questions, les passages "moléculaires », les chaînons reliant l'idée à la réalisation, et donc tout aussi bien la clarté des idées, font cruellement défaut. On peut légitimement se demander si la prolifération d'une discursivité «radicale» et postmarxiste faite de débris et de morceaux de pensées politiques n'aurait pas pour effet d'occulter la contingence et la précarité à laquelle renvoient ces questions, et de refouler le fait que les pratiques militantes, et toute politique authentique, appellent moins le foisonnement explosif du Savoir que sa destitution, moins la multiplication frénétique des mots et des discours que l'attention aux silences creusés par toute réelle "prise de parole ». Le réel politique est toujours un critère de sélection et de raréfaction.

\section{$\mathbf{X I}$}

58 Il est certainement présomptueux de partir d'une expérience limitée - toutes les expériences le sont par définition - pour tirer des conclusions aussi massives sur l'état de l'époque présente ; et surtout de croire que ces questionnements et ces difficultés ne font pas déjà l'objet des réflexions et des préoccupations de nombreux acteurs des « lieux communs d'humanité ». 
59 Mais il ne s'agit nullement de nier l'existence de ces réflexions, ni de supposer que les militants et les activistes des « bonnes pratiques » n'aient pas une conscience claire ni un discours lucide sur ce qu'ils font et sur les limites de leur action. Mais justement, il s'agit de reconnaître les dispositifs, les pièges idéologiques, les régressions non innocentes, qui empêchent cette conscience et cette réflexion de produire tous les effets dont elles sont capables à une échelle de moins en moins restreinte. Et la seule bonne raison de tenter quelques généralisations sur de tels sujets est l'expérience quotidienne de l'inadéquation entre sa propre conduite et l'effort requis pour déployer ces effets critiques.

Finalement, il ne s'agit point de disqualifier ces actes, ni de légiférer sur les conduites militantes, mais de voir en quoi certains phénomènes que l'on pourra qualifier d'« intellectuels» et un certain rapport à la fonction intellectuelle en tant que telle facilitent ou entravent le travail immense et anonyme des différents «lieux communs d'humanité »- puisqu'il est évident que le contexte dans lequel est inscrite la réflexion que nous livrons au lecteur fait qu'elle relève immédiatement des préoccupations, des habitus et des référentiels de la couche spécifique des intellectuels tels que les institue la division du travail. La question est donc aussi celle des insuffisances des intellectuels devant ce qui se passe au niveau des pratiques politiques ou pré-politiques : ce n'est peutêtre pas la question en soi décisive, mais c'est, pour nous, le point de départ immédiat et « naturel » qui permet d'explorer des enjeux plus vastes.

Cette insuffisance tient, lorsqu'il est question de la discursivité généralisée, à la fonction affirmative de la culture dans sa manifestation la plus déployée, à savoir la philosophie.

Dans un commentaire de Lacan, Alain Badiou a résumé l'opposition entre l'opération philosophique et l'acte analytique tels que Lacan les conçoit :

Les opérations philosophiques prétendent ultimement délivrer une satisfaction, voire une béatitude (...). Il est de l'essence de l'activité philosophique d'énoncer que son produit propre est la possibilité d'une béatitude intellectuelle et d'en examiner le prix. On pourra dire que, du point de vue de son acte, le sujet philosophique se présente comme un sujet virtuellement comblé (...). Au contraire, l'acte analytique, pour le psychanalyste lui-même, ne suscite que l'angoisse et le malaise ${ }^{39}$.

62 Ce qui est en cause ici est un certain pouvoir de synthèse de l'acte philosophique, qui tend à présenter toute détermination du réel comme un moment inscrit dans le réseau des enchaînements conceptuels tels que pourrait les déployer un discours «total ». Cette tentation synthétique est peut-être un autre avatar de la tendance à esquiver la confrontation avec un réel hétérogène et dérangeant qu'on a repérée au cœur des mises en scène, des spectacles et en général de toutes les formations mélancoliques ${ }^{40}$. Cette « sagesse » qui prétend incarner l'immanence de la réalisation dans la congruence d'une Théorie totale et d'un Ens realissimum est donc la mélancolie suprême, si l'on considère comme mélancolique toute tendance à détourner les "possibilités spirituelles de l'homme » d'une confrontation avec un Autre réel. Ce que Lacan qualifie de "discours philosophique » est en réalité l'opération d'un discours sursaturé, dont l'efficacité ne sort pas du cercle magique du travail de soi sur soi.

Il y a eu au contraire, dans l'histoire des séquences politiques, des opérations dans le savoir plus proches de ce que Lacan entend par "acte analytique». Par exemple, la diffusion de la pratique de l'enquête en Italie à partir des années 1950. Dans et par ces enquêtes menées dans le Sud rural, dans les banlieues du Nord de l'Italie, dans les grandes usines des villes industrielles, des intellectuels comme Raniero Panzieri et Ernesto de Martino ont rompu avec les certitudes lénifiantes de discours non moins synthétiques 
que la philosophie politique post-marxiste - le matérialisme dialectique, l'historicisme de Benedetto Croce, le gramscisme de Togliatti - et ont inventé un autre rapport entre le savoir et la politique, un rapport dans lequel l'interruption de la parole savante, son articulation au silence et à d'autres paroles et à d'autres gestes, comptaient davantage que sa continuité totalisatrice.

C'est l'exemple d'une pratique intellectuelle qui se construit autour de la déstabilisation du discours autocentré de la philosophie-politique: un savoir qui n'est énonçable que depuis un lieu " autre ", décentré, toujours-déjà en train d'exploser vers les choses...

\section{XII}

Qu'il me soit permis d'évoquer une dernière fois des souvenirs personnels. Ceux qui ont pu rencontrer les derniers feux du militantisme traditionnel dans l'Italie des années 1990, juste après la dissolution de l'URSS, peuvent témoigner du remplacement progressif de la passion du réel par l'attachement aux simulacres. Dans des milieux liés à des paradigmes marxistes relativement traditionnels, hérités du PCI ou de certains secteurs de la Nuova Sinistra, tels qu'on pouvait les trouver dans un parti comme Rifondazione Comunista, l'usage des références classiques - les œuvres de Marx et de Lénine, la "centralité ouvrière ", voire même des outils théoriques d'une extrême complexité, telle la théorie de la valeur - était déjà largement décalé par rapport aux pratiques militantes, et n'était plus qu'un usage, comme l'aurait dit Elio Vittorini, "consolateur», ne visant que la réaffirmation des raisons de ne pas céder, de tenir individuellement et collectivement devant les terribles démentis infligés par l'histoire. En ce qui concerne les mouvances héritant de l'Autonomie des années 1970, elles étaient fortement tentées par la constitution de communautés séparées - les "oasis ", déjà - et par la beauté de l'acte gratuit lors des manifestations, fidèles en cela à l'un des traits les plus douteux des mobilisations de 1977.

Il serait sans doute injuste de réduire ces expériences à des aspects qui étaient des conséquences probablement inévitables de la phase historique, laquelle imposait de rechercher rapidement des stratégies de survie devant l'effondrement de la perspective communiste et la triste euphorie restauratrice qui traversait le monde de l'après-Mur de Berlin. Mais il est impossible de ne pas constater que les symptômes de l'impasse qui se manifestaient déjà vers 1994 ont été ratifiés et généralisés par l'histoire ultérieure, par l'impossibilité de redonner vie à un processus politique effectif.

Il y avait pourtant autre chose à cette époque-là, qui ne devrait pas être oublié. C'était la capacité, qui subsistait dans ces contextes militants, d'appliquer un effort collectif à un projet réfléchi, avec ses étapes nécessaires et ses conditions indispensables ; c'était donc la capacité de maîtriser de telles étapes et conditions, et l'expérience de la joie liée à cette maîtrise et à son apprentissage. La capacité et la joie de mesurer ses forces et ses désirs au travail requis par une réalisation, bien qu'à une échelle désespérément insuffisante par rapport à la réalité générale des rapports sociaux.

C'était aussi la conviction qu'un tel travail était intrinsèque à la pratique militante, que c'était finalement quelque chose de tout-à-fait normal d'accéder à des savoir-faire qui demandaient certes l'effort d'un apprentissage, mais qui étaient entièrement le fait d'individus ordinaires, à la portée de n'importe quel homme ou femme ayant fait le choix de militer. 
69 Il y a quelque chose de précieux, et qui permettrait de rouvrir tout ce discours, dans ce refus du privilège accordé à des moments, à des gestes ou à des paroles prétendument exceptionnels, dans cette distance prise à l'égard du primat de l'irruption verticale, extatique et $«$ spectaculaire $»^{41}$.

La possibilité de ne pas se contenter de mises en scène, de spectacles et de discours philosophiques, nous la devrons peut-être à la réactivation de cette capacité à supporter la contradiction et la négativité tout en les inscrivant dans les contextes les plus ordinaires, dans la durée anonyme des gestes et des vies. C'est le lieu où devrait se faire la séparation, l'ana-lysis, à l'égard des fausses images du Bien. C'est peut-être cette articulation de l'absolu et de l'ordinaire qui pourra dissiper la mélancolie et ses fantasmes, et nous faire finalement accéder à des formes et à des rites qui, au lieu d'éloigner la confrontation avec l'altérité et la contingence, n'existeraient que pour les accueillir et les faire résonner.

Car les pratiques militantes et l'engagement ne peuvent éviter d'incorporer une dimension formelle, dont la « professionnalisation » et les compétences quasi-spécialisées sont le versant pragmatique qui contient et soutient en même temps leur prégnance symbolique et rituelle. Entretenir le lien entre principes et conséquences; opérer la synthèse entre le projet et son actualisation : tout cela permet de mener efficacement une action transformatrice, et fournit en même temps au sujet de cette action le modèle d'une consistance éthique qui repose sur l'épreuve d'une telle efficacité.

Les formes efficaces de cette action sont à la fois une force matérielle de transformation du réel et la base réelle d'une intensification de la vie de l'âme. Toute la difficulté consiste à faire en sorte que la consistance de ces formes ne se réduise pas à une compensation, à une jouissance immanente, mais laisse entrevoir ses propres lacunes et indique, au-delà des formes et de leurs lois intrinsèques, le réel qu'elles cachent et révèlent :

Si l'on cessait de rechercher en nous-mêmes et de proposer à autrui un centre " authentique ", la possibilité resurgirait de vivre simultanément dans la vérité et dans la non-vérité, d'être et de ne pas être au même moment, de participer et de s'éclipser (...). Il est impossible d'être dans le monde sans être du monde. Mais, si l'on pouvait conférer à son existence, individuelle ou collective, un maximum de ritualité (donc un maximum de renoncement à l'authenticité et un maximum de formalisation), alors l'exercice d'une vie rituelle témoignerait d'un centre vide (...). L'expérience d'un centre (...) conduit à attribuer une valeur immense aux comportements pratiques (...), donc à la «vie » quotidienne précisément dans la mesure où elle peut être vécue comme l'envers d'une Altérité absolue qui l'accompagne, à l'instar de l'ombre qui accompagne la lumière et vice versa (...). Par sa formule du "chevalier de la foi ", Kierkegaard visait à fournir un modèle susceptible d'unir la «démocratie» de la vie quotidienne avec l'«aristocratie » qu'est l'unicité de la vie de l'individu. Car ce n'est pas vrai que «la vraie vie est ailleurs ». Il n'y a aucune vraie vie. La seule vie moins fausse est une vie qui ne recherche pas le centre parce qu'elle sait qu'un tel centre existe et qui travaille au contraire sur ces décentrements susceptibles de transférer plus d'énergie au système et donc de le déséquilibrer. C'est aussi le sens de l'histoire du « fils de Dieu », qui, pour pouvoir être un homme, a dû se diviser d'avec le Père ${ }^{42}$. 


\section{NOTES}

1. Jacques Rancière, En quel temps vivons-nous? Conversation avec Eric Hazan, Paris, La Fabrique, p. 22-23.

2. Ibid., p. 23.

3. Ibid., p. 27-28.

4. Ibid., p. 28-29.

5. Cette notion d'un «peuple absent » est centrale dans la lecture que Gilles Deleuze donne du cinéma de Jean-Marie Straub et Danièle Huillet . L'absentement d'un sujet collectif de l'émancipation dans certaines séquences historiques, voire l'absence de perspectives politiques dans de telles séquences, peut bien s'exprimer dans une œuvre artistique sous la forme de l'absence du peuple. Mais une telle catégorie ne saurait faire l'objet d'un usage directement politique : l'impasse réelle que cache (et révèle) l'idée de l'absence d'un peuple demande une autre manière de déterminer l'objet. Dans l'art il y a toujours un moment d'idolâtrie : pour retrouver le réel qu'il exprime, il faut briser les images. Sur ces points je me permets de renvoyer à Andrea Cavazzini, « "Des choses très anciennes mais oubliées". Notes sur Jean-Marie Straub et Danièle Huillet », in Cahiers du GRM, 8, 2015, OpenEdition https://journals.openedition.org/ grm/739\#ftn4.

6. Giorgio Agamben, Stanze, traduit de l'italien par Yves Hersant, Paris, Rivages, 1994, p. 48.

7. Ibid., p. 47.

8. Ibid., p. 49.

9. Ibid., p. 26.

10. Herbert Marcuse, «Réflexions sur le caractère "affirmatif" de la culture » (1937), dans Id., Culture et société, traduit par G. Billy, D. Bresson et J.-B. Grasset, Paris, Minuit, 1970, p. 116-118.

11. Antonio Gramsci, Passato e presente, Rome, Editori Riuniti, 1975, p. 5-6.

12. Ibid., p. 6.

13. Max Weber, «La profession et la vocation de politique », in Le savant et le politique, traduit et présenté par Catherine Colliot-Thélène, Paris, La Découverte, 2003, p. 138-139.

14. Ibid., p. 137.

15. Ibid., p. 150.

16. Ibid., p.

17. Jules Andrieu, Notes pour servir à l'histoire de la Commune de Paris en 1871, Clermont-Ferrand, Libertalia, 2016, p. 235.

18. De toute évidence, la recherche systématique de l'affrontement direct avec les forces de police, loin de protéger physiquement les manifestants, contribue à la banalisation de l'extrême violence dont la police use de plus en plus à leur égard. Cette spirale mortifère n'est pas une nouveauté : à une échelle bien plus importante et tragique, elle a dominé la dynamique de la lutte armée dans les années 1970.

19. Jean -Paul Sartre, Esquisse d'une théorie des émotions, Paris, Hermann, 1995, p. 79.

20. op. cit., p. 81-82.

21. Franco Fortini, "Per una congiura in piena luce ", in Saggi ed epigrammi, a cura di Luca Lenzini, Milan, Mondadori, 2003, p. 1053.

22. Ibid., p. 1052.

23. Ibid., p. 1053.

24. J. Rancière, En quel temps vivonsnous?, op. cit., p. 37 
25. Enrico Donaggio, Dire di no, Milan, Feltrinelli, 2016. Voir aussi A. Cavazzini, « Mémoire posthumes du communisme", in Cahiers du GRM, 12, OpenEdition, 2017, https:// journals.openedition.org/grm/923

26. Nathalie Quintane, Un œil en moins, Paris, P.O.L., 2018, p. 273.

27. Ibid., p. 272.

28. Pour se limiter à l'exemple le plus immédiat, il me semble incontestable que la «guerre contre les migrations » est un enjeu central dans le «cycle politique réactionnaire ouvert par et dans la crise européenne " (Giso Amendola, «Il ministro dei confini e quell'insopportabile indisciplina degli avvocati », in Euronomade, 15/06/2018, http://www.euronomade.info/?p=10768 ), ce qui rend, non fausse, mais sans doute insuffisante, sa caractérisation en termes de «diversion » mise en œuvre par les dirigeants européens pour détourner l'attention des luttes sociales (cf. dans ce Cahier, l'entretien avec Xavier Vigna). Cela signifie en tout cas que l'action d'associations et collectifs engagés sur ce front trouve de marges de manœuvre de plus en plus étroites et difficiles pour obtenir des résultats pratiques. Il est en tout cas significatif que les pratiques les plus efficaces dans ce domaine supposent une certaine compétence spécialisée, notamment juridique, de la part des acteurs.

29. Walter Benjamin, «Commentaires des poèmes de Brecht » (1938-1939), in CEuvres III, trad. fr. de M. de Gandillac, R. Rochlitz et P. Rusch, Paris, Gallimard, 2000, p. 267-268. Le poème auquel fait allusion Benjamin a été traduit par Guillevic in B. Brecht, Poèmes I, Paris, L'Arche, 1965, p. 58.

30. Jean Oury, Il, donc, Paris, 10/18, 1978, p. 78-79.

31. Marco Revelli, « Il voto come un'antica festa crudele. Vince la cattiveria », dans Il Manifesto du 27/06/2018.

32. J. Rancière, En quel temps vivons-nous?, op. cit., p. 12-13.

33. Ibid., p. 38.

34. Ibid., p. 12.

35. Ibid., p. 21.

36. Ibid., p. 20.

37. Ibid., p. 38.

38. Alessandro Fontana, "Lire Foucault, aujourd'hui », in L'exercice de la pensée. Machiavel, Leopardi, Foucault, Paris, Publications de la Sorbonne, 2015, p. 286.

39. Alain Badiou, Le Séminaire. Lacan. L'antiphilosophie 3. 1994-1995, Paris, Fayard, 2013, p. 168.

40. À très juste titre, un autre commentateur de Lacan a dit que, pour le psychanalyste français, « les organisations politiques dont le but est de durer [en tant que collectifs imaginaires] sont en réalité des organisations philosophiques» (Bruno Moroncini, Lacan politico, Naples, Cronopio, 2014, p. 18-19). Le glissement des pratiques militantes vers des pratiques imaginaires opérant un effet de groupe est donc aussi un glissement vers l'absorption de la politique dans la philosophie en tant que discours du Tout-Savoir : c'est la situation de la Gauche hégélienne avant la rupture opérée par Marx et Engels...

41. Gramsci l'avait déjà dit, en stigmatisant la tendance à identifier révolution et enthousiasme: «L'enthousiasme n'est que l'adoration extérieur d'un fétiche (...). Le seul enthousiasme justifiable est celui qui accompagne la volonté intelligente, l'ardeur intelligente au travail, la richesse d'inventions dans les initiatives concrètes qui modifient la réalité donnée » (A. Gramsci, Passato e presente, op. cit., p. 9-10).

42. F. Fortini, Extrema ratio, Milan, Garzanti, 1994, p. 96-98. 


\section{RÉSUMÉS}

L'article porte sur les racines du rapport contemporain au passé révolutionnaire

INDEX

Thèmes : philosophie, histoire, politique

Index chronologique : XXème siècle, XXIème siècle

Index géographique : Europe occidentale

Mots-clés : mélancolie, révolution, militantisme, black bloc, Agamben Giorgio, Rancière Jacques, Fortini Franco, communisme

\section{AUTEUR}

\section{ANDREA CAVAZZINI}

Agrégé de philosophie, membre du GRM 